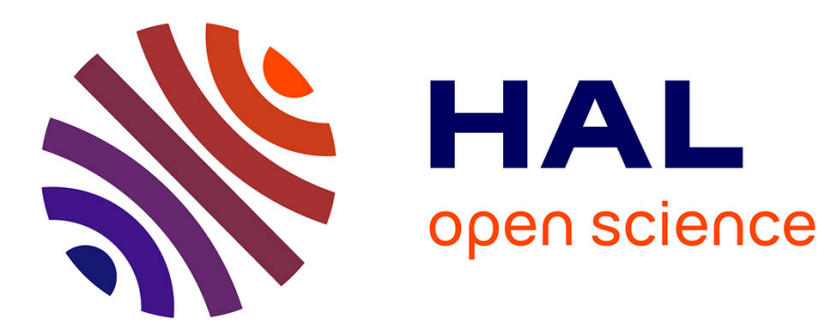

\title{
Un morceau préservé de la flore provençale
}

René Molinier

\section{To cite this version:}

René Molinier. Un morceau préservé de la flore provençale. Revue forestière française, 1971, 23 (S), pp.184-189. 10.4267/2042/20562 . hal-03395043

\section{HAL Id: hal-03395043 \\ https://hal.science/hal-03395043}

Submitted on 22 Oct 2021

HAL is a multi-disciplinary open access archive for the deposit and dissemination of scientific research documents, whether they are published or not. The documents may come from teaching and research institutions in France or abroad, or from public or private research centers.
L'archive ouverte pluridisciplinaire HAL, est destinée au dépôt et à la diffusion de documents scientifiques de niveau recherche, publiés ou non, émanant des établissements d'enseignement et de recherche français ou étrangers, des laboratoires publics ou privés. 


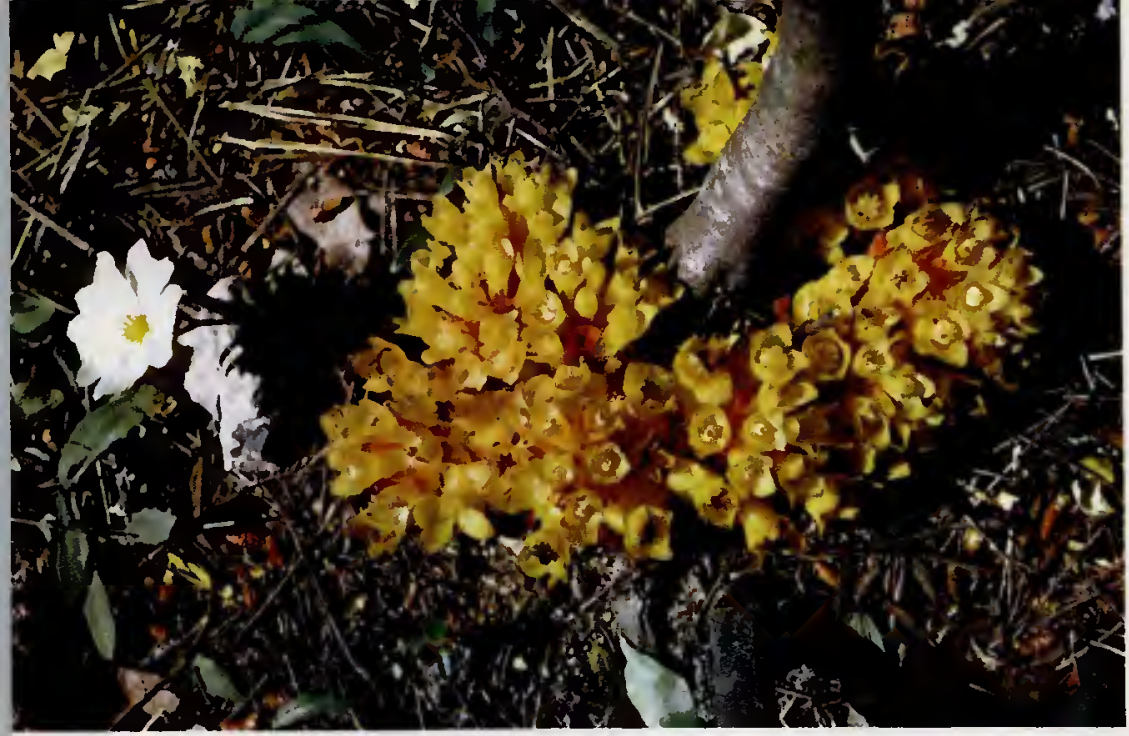

Cytinet parasitant des Cistes

Photo FISCHESSER

Fleurs do Mesembrianthénum ou Griffes de Sorcières

Photo FISCHESSER
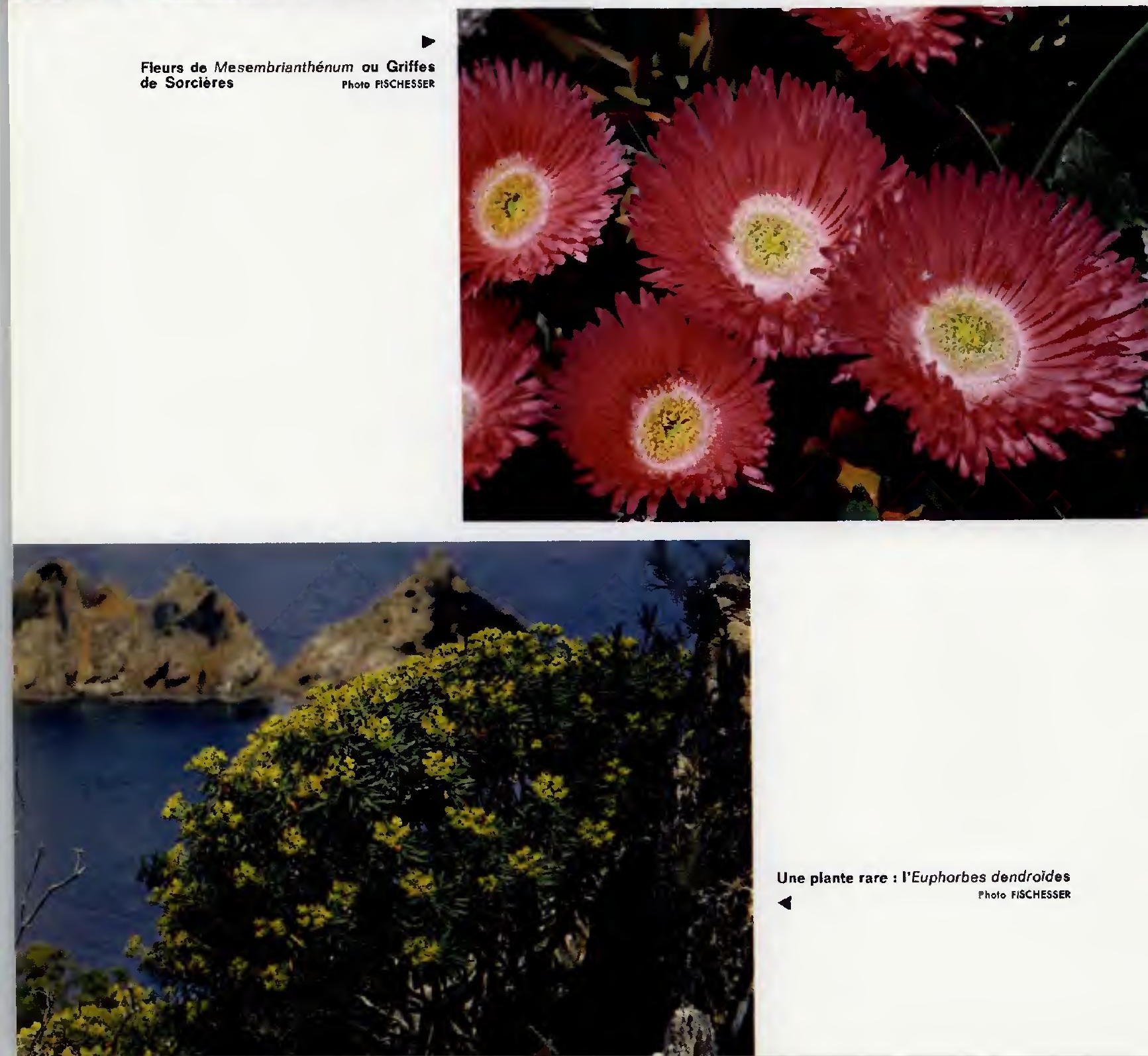


\section{UN MORCEAU PRÉSERVÉ DE LA FLORE PROVENÇALE}

R. MOLINIER

Class. Oxford $(44 \times M 2-57 L) 174: 907.12$

Depuis longtemps, et d'un mouvement accéléré au cours de ces dernières années, la pression touristique sur les rivages ensoleillés de la Méditerranée, s'accompagne inéluctablement d'un accroissement constant des risques d'incendie; les événements de l'automne 1970 ne l'ont malheureusement que trop confirmé et d'une manière tristement spectaculaire.

II est donc grand temps de protéger certains sites représentatifs d'une végétation que menace une ruine prochaine. L'urgence d'une protection efficace se fait notamment sentir dans la zone littorale depuis le Rhône jusqu'à la frontière italienne et au-delà, et surtout en Provence cristalline, Maures-Esterel. Pour la Provence calcaire, particulièrement aride, autour des grandes cités que sont Marseille et Toulon, la sylve littorale a d'ores et déjà disparu; il n'y reste guère plus de territoire susceptible d'être englobé dans un parc national sinon la Camargue, d'un intérêt international par l'accueil qu'elle assure aux grandes migrations d'oiseaux. Encore peut-on se demander ce qu'il adviendra de cette terre d'exception, prise dès maintenant dans l'étau des aménagements du Languedoc-Roussillon à l'Ouest, du complexe de Fos à l'Est, lorsque le grand axe économique Rhin-Méditerranée sera pleinement réalisé ! Jusqu'à présent la Provence cristalline a conservé - bien qu'altéré en de nombreux points une grande partie de son capital forestier parce qu'elle est plus éloignée des grands centres urbains, les grands axes de communication contournant les Maures par la dépression permienne de Cuers - par ailleurs plus accessible aux cultures que les reliefs cristallins - et évitant le littoral tourmenté de l'Esterel.

D'autre part la végétation, en Provence cristalline, résiste mieux au feu qu'en Provence calcaire. Les phyllades, gneiss et micaschistes qui la constituent, facilement délitables sous l'effet de prècipitations atmosphériques assez élevées $(700$ à $1000 \mathrm{~mm}$ contre 500 à 700 en Provence calcaire littorale) facilitent la reprise de la végétation naturelle après le passage du feu auquel, par ailleurs, le Chêne-liège résiste mieux que le Chêne vert.

La végétation de la Provence cristalline sera de ce fait sensiblement différente de celle de la Provence calcaire. Pin maritime surtout et Pin pignon y remplacent le Pin d'Alep; le Chêne-liège $y$ domine le Chêne vert; les maquis à Ericacées s'y substituent aux garrigues à Chêne kermès ou Romarin; et il n'est pas jusqu'aux pelouses à Hélianthèmes - stades ultimes de dégradation de la forêt - quil n'y soient très différentes des " bauques " à Brachypodes. 
Mais dẻlimiter un parc national ou régional dans une partie des Maures et de l'Esterel est délicat; non qu'il ne s'y trouve des zones d'un trẻs grand intérêt - citons seulement les magnifiques forêts du Dom, de Lambert, de la Verne entre tant d'autres -, mais on aime à penser d'abord à des ensembles bien isolés du reste du tapis végétal presque immanquablement voué aux prochains incendies. Les îles d'Hyères, justement nommées pour leur beauté, les "lles d’Or " et parmi elles surtout Port-Cros, étaient toutes désignées pour la création d'un parc national. Pourquoi plus particulièrement Port-Cros ? Parce que depuis longtemps protégée par Marcel Henry et sa femme, propriétaires avisés et attentits d'une importante partie de l'île, elle a échappé à la fois à l'Incendie et à une colonisation touristique abusive.

Ce n'est pas que sa végétation présente une richesse exceptionnelle. Avec à peine plus de 530 espèces de Spermaphytes (l'ensemble des Stoechades en compte près de 750 et le département du Var près de 2200 ), sa flore est moins riche que celle des Maures voisines ; on n'y voit pas l'endémique Delphinium requieni de Porquerolles; et si le Teucrium marum y est commun, on n'y voit pas davantage le Teucrium massiliense et l'élégant Cirsium trispinosum de l'île du Levant, les trois dernières espèces rappelant la Corse - tout comme le rare Artemisia arborescens du château du Fort du Moulin -. Quant au magnifique Euphorbia dendroides de l'Estissac, il n'est point rare sur le littoral voisin.

Mais, contrairement aux massifs forestiers qui lui font tace sur le continent et que le feu ravage périodiquement, cette végétation, si elle n'est pas réellement riche a pu évoluer librement presque depuis le début de notre siècle ; la couverture végétale se montre donc à PortCros en grande partie parvenue aux termes ultimes de l'évolution progressive : haut maquis et forêt climacique.

On sait que l'évolution naturelle, à partir du sol nu, comporte en terrain acide la succession classique: pelouse à Hèlianthèmes, Cistaie, maquis bas à Bruyère à balais et Lavande des Maures, maquis élevé à Bruyère arborescente et Arbousier, forêt de Chêne-liège ou de Chêne vert. Effectivement, seuls les stades ultimes de cette série sont actuellement représentés à Port-Cros, tant le retour aux climax y est avancé. On ne voit plus la pelouse qui s'égrène discrètement sur le bord des sentiers ou chemins bien dẻgagés; la Cistaie ne s'observe guère qu'autour des forts - oủ les destructions ont été particulièrement intenses - ou sur les ultimes zones cultivejes de la Solitude et la Palu; le maquis bas est pratiquement absent dans l'île; et si le maquis élevé à Bruyère arborescente et Arbousier y est encore étendu il y atteint souvent la valeur du stade forestier (6-8 mètres de hauteur vers la Palu), et la fréquence des Chênes qui le parsèment en de nombreux points y garantit la prochaine réalisation de la Chênaie climacique.

De quelle Chênaie s'agit-il ? Suberaie ou Chênaie d'Yeuses ? Les études phytosociologiques que j'ai consacrées à l'ensemble Maures-Esterel, surtout l'étude des cartes au $1 / 20000^{\text {e }}$ très fouillées que j'ai faite de leur végétation montrent que la Suberaie y domine largement la Yeuseraie ; pourquoi cette Yeuseraie est-elle donc à nos yeux le climax plutôt que la Suberaie?

Remarquons d'abord que la Yeuseraie, si elle recherche les fonds de vallons plus humides sur le continent, n'y est nullement exclue du littoral immédiat; on l'y voit par exemple immèdiatement en arrière de la zone halophile et du liseré formé par la brousse à Oléastre et Lentisque au cap Siciè, sur la presqu'île de Giens, aux caps de Léoube et Nègre, et elle m'a paru partout possible en Provence cristalline au méme titre que la Suberaie.

Par ailleurs, partout où je les ai vus en concurrence, le Chẻne vert m'a paru prendre l'avantage sur le Chêne-liège; ce dernier, en effet, contrairement au Chêne vert, exige un couvert clairsemé ; un couvert dense tel que peut le former le Chêne vert interdit donc le Chêne-liège alors que le Chêne vert n'est nullement exclu du couvert des Chênes-liège. Dans ces conditions l'issue de leur lutte possible ne peut être que favorable au Chêne vert.

Notons encore que les parties de la Provence cristalline où les circonstances ont permis l'évolution naturelle prolongèe de la vègétation sont partlculièrement instructives. Or à Port-Cros depuis longtemps protẻgé des destructions, à Porquerolles où une exploitation modérée jusqu'à nos jours a conservé de magnifiques taillis, sur l'isthme de Giens qui ne date que de quelques siècles avant notre ère : c'est le Chêne vert qui s'est installé, non. le Chêne-liège ; l'inverse ne se serait-il pas manifesté si la forêt originelle avait été la Suberale?

Enfin soulignons que le Chêne-liège, même à l'état isolé, est rare à Port-Cros et s'observe presque uniquement en bordure d'anciennes cultures dans le vallon de la Solitude, autour de la 


\section{CARTE DES ASSOCIATIONS VÉGÉTALES DE L'ILE DE PORT-CROS}
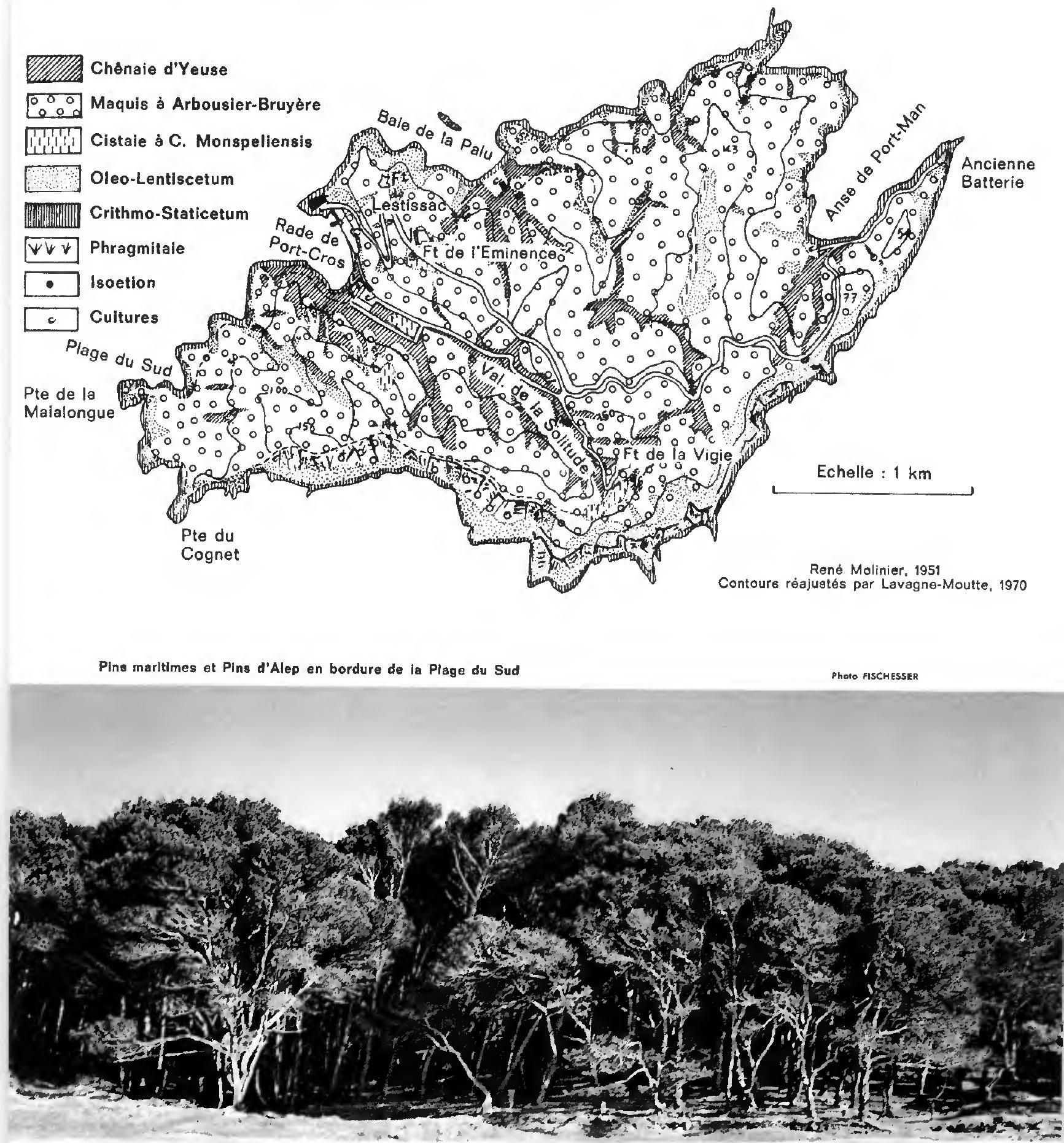
Sardinière longtemps habitée ou en quelques autres points ouverts au passage de l'Homme comme si ce dernier l'avait localement favorisé.

Tout concourt ainsi à déterminer ma conviction que le climax est bien la Chénaie d'Yeuses.

C'est le Quercefum ilicis des phytosociologues, floristiquement assez pauvre car il fut un temps où, décimée par l'homme, la Chênaie ne devait exister qu'en quelques refuges rocheux inaccessibles aux cultures. La Yeuseraie se présente souvent, à Port-Cros, sous la forme d'un taillis quasi pur de Chênes verts dont le sous-bois, plus ou moins clairsemé, est encore formé par les espèces du maquis; parfois s'y observent des caractéristiques du sous-bois de la Yeuseraie : le Petit Houx, le Carex distachya, la Capillaire noire (Asplenium adianthum nigrum), quelques arbustes (Phillyrea media et angusfitolia, Rhamnus alaternus) et l'une ou l'autre ou pusieurs des lianes habituelles à ce type de forêt sempervirente : Clématite brûlante. Salsepareille, Garance voyageuse, Asperge sauvage.

Selon que l'on reconnaîtra déjà l'association du Chêne vert dans le taillis pur ou que l'on exigera d'y voir en sous-bois quelques-unes des caractéristiques ci-dessus, l'importance attribuée à la Yeuseraie de Port-Cros sera plus ou moins grande. C'est la première solution que j'ai adoptée sur la carte au $1 / 25000^{\circ}$ de l'île publiée dans cette Revue même, en 1952, car je voulais souligner l'importance édificatrice du Chêne vert pour le plein développement de l'association à laquelle il donne son nom; c'est la seconde solution qu'adoptent mes collaborateurs le Professeur Lavagne et $A$. Moutte pour une carte détaillée à très grande échelle $\left(1 / 5000^{e}\right)$ levée dans le cadre des travaux entrepris par le Comité scientitique du Parc de PortCros, et dont figure ici une réduction simplifiée au $1 / 25000$. L'exigence de départ, dilférente, explique l'importance moins grande donnée au Quercetum ilicis par mes collaborateurs.

Mais de toute manière il apparaît que la Yeuseraie est en bonne voie de reconstitution sur PortCros, alors qu'à la fin du $19^{\circ}$ siècle encore, cette île, largement cultivée, n'y conservait que quelques îlots de Chênaie, et que la très vieille carte de Cassini la figurait dépourvue de tout élément forestier

Et les Pins? nous demandera à juste raison le lecteur de ces lignes. Car, d'où que l'on aborde à Port-Cros - sauf du côté sud, mais qui se risquerait à y accoster ? - c'est une forêt de Pins d'Alep qui paraît recouvrir entièrement l'île. Effectivement, c'est seulement la zone fortement battue par les embruns - domaine du Statice et du Crithmum -, la partie occidentale fortement balayée par un mistral imposant au Chêne comme aux arbustes du maquis un port couché caractéristique, qul sont évitées par le Pin d'Alep. Pratiquement ce Pin forme une strate forestière supérieure quasi continue dans l'île (quelques îlots seulement sur Bagaud). On remarquera cependant que les sujets jeunes sont rares et que beaucoup de vieux Pins, morts de vieillesse, ne laissent aucune descendance : le Pin d'Alep, essence de lumière, ne peut pousser dans la Chênaie ou le maquis denses; il a colonisé l'île à une époque où, la végétation naturelle étant détruite, il a pu prendre pied et pousser plus rapidement que les Chênes. II n'a plus sa place que pour un temps au-dessus des taillis et hauts maquis; dans les conditions naturelles de l'évolution, il est appelé à perdre de l'importance à PortCros et à se localiser en quelques points rocheux impropres au développement d'une végétation dense et continue (côte sud par exemple).

Nous ne signalerons que pour mémoire les rares Pins maritimes que l'on voyait, disséminés un peu partout, surtout à la Sardinière et à la Plage du Sud: ils n'ont pas résisté à la cochenille dont chacun connaît la triste et récente action destructrice dans toute la Provence cristalline.

L'îlot voisin de Bagaud comporte, comme Port-Cros, en arrière et au-dessus de la zone halophile, une ceinture d'Oleo-Lentiscetum, entourant un maquis à Bruyère arborescente et Arbousier d'où émergent quelques bouquets de Pins d'Alep. Ces deux ceintures sont surtout bien développées sur la côte occidentale, c'est-à-dire face au mlstral. Soulignons l'absence totale des Chênes qui ont sans doute été éliminés de Bagaud par les tirs de la marine mais qui s'y développeraient dans le maquis si des glands y étaient introduits.

Ce court aperçu sur la végétation de Port-Cros, s'il souligne l'intérêt de la Chênaie et du haut maquis qui la précède, serait incomplet s'il n'était tait mention, sinon de certaines associations végétales qui intéressent surtout les botanistes (associations halophiles à Crithmum et Sfatice, à Anthyllis barba-jovis et Planfago subulata, pointements de l'association à /soetes durieui), du moins d'un second groupement climacique réduit à une frange étroitement littorale et discontinue en certains points : la brousse à Oléastre et Lentisque, avec le Myrte et un 
Genévrier rouge (1). Entre la zone halophile et le maquis - de $2-3 \mathrm{~m}$ à $6-8 \mathrm{~m}$ d'altitude, davantage face au mistral - ce groupement se reconnaît à l'allure brossée, dense et luisante du peuplement impénétrable qu'il forme. If est surtout remarquable entre la Palu et le Fort de l'Estissac où l'accompagne une remarquable caractéristique de l'Oleo-Lentiscetum: Euphorbia dendroides. Cet Euphorbe, abondant sur la Cóte d'Azur entre Nice et Menton, se raréfie dans les Maures oú il atteint sa limite occidentale à Port-Cros et, en face, à SaintClair et au Lavandou (si l'on fait abstraction des environs d'Hyères où il a sans doute été introduit).

En somme, sans être d'une richesse exceptionnelle, la végétation du Parc national de PortCros est un bon spécimen de la végétation propre à la Provence cristalline; elle présente à Port-Cros un état d'évolution vers le climax d'autant pus remarquable et précieux que la sylve continentale voisine est plus menacée dans son existence par le feu et le développement de l'urbanisme sur nos côtes.

Si l'on considère en outre sa protection facilitée par l'isolement en mer il apparaît bien que Port-Cros ètait particulièrement désignée pour une réserve intéressant la Provence cristalline.

Mais pour que la sollicitude des hommes s'exerce sur un ensemble plus complet de la flore et de la végétation de cette région, il conviendra d'adjoindre un jour au Parc national de PortCros certains secteurs des Maures, par exemple les forêts du Dom, de la Verne, du plateau Lambert ou de Notre-Dame des Anges.

(1) II s'agit d'une forme tout à fait littorale du Genévrier rouge, dont LINNE faisait une espèce distlncte (Juniperus lycla) et que R. MAIRE, dans sa Flore de l'Afrlque du Nord, consídère comme une simple forme : $J$. phoenleea L. var. eu-phoenlcea Malre et Weller, forma mollis $M$. et $W$. Ce Genévrler littoral, qui peut ailleurs devenir arborescent, a un port très particulier, pyramidel dans le jeune âge avec des rameaux souvent horizontaux, des baies plus grosses a nucules molns nombreux que chez le type de l'intérieur.

\section{René MOLINIER}

Professeur honoraire a le Faculté des sciences de Marseille

Place Victor-Hugo

13 - MARSEILLE $\left(3^{\circ}\right)$ 